\title{
Confinement of Positrons Exceeding One Second in a Supported Magnetic Dipole Trap
}

\author{
J. Horn-Stanja, ${ }^{1, *}$ S. Nißl, ${ }^{1,2}$ U. Hergenhahn, ${ }^{1,3}$ T. Sunn \\ Pedersen, ${ }^{1,4}$ H. Saitoh, ${ }^{1,5}$ E. V. Stenson, ${ }^{1,2,6}$ M. Dickmann, ${ }^{2,7}$ C. \\ Hugenschmidt, ${ }^{2,7}$ M. Singer, ${ }^{2,7}$ M. R. Stoneking, ${ }^{8}$ and J. R. Danielson ${ }^{6}$ \\ ${ }^{1}$ Max Planck Institute for Plasma Physics, \\ Garching and Greifswald, Germany \\ ${ }^{2}$ Technische Universität München, Garching, Germany \\ ${ }^{3}$ Leibniz Institute for Surface Engineering (IOM), Leipzig, Germany \\ ${ }^{4}$ University of Greifswald, Greifswald, Germany \\ ${ }^{5}$ The University of Tokyo, Kashiwa, Japan \\ ${ }^{6}$ University of California, San Diego, La Jolla, CA, USA \\ ${ }^{7}$ Heinz Maier-Leibnitz Zentrum (MLZ), Garching, Germany \\ ${ }^{8}$ Lawrence University, Appleton, WI, USA
}

(Dated: February 13, 2019)

\begin{abstract}
An ensemble of low-energy positrons injected into a supported magnetic dipole trap can remain trapped for more than a second. Trapping experiments with and without a positive magnet bias yield confinement times up to $\tau_{A}=(1.5 \pm 0.1) \mathrm{s}$ and $\tau_{B}=(0.28 \pm 0.04) \mathrm{s}$, respectively. Supported by single-particle simulations, we conclude that the dominant mechanism limiting the confinement in this trap is scattering off of neutrals, which can lead to both radial transport and parallel losses onto the magnet surface. These results provide encouragement for plans to confine an electron-positron plasma in a levitated dipole trap.
\end{abstract}


A magnetic dipole field can confine charged-particle ensembles with an arbitrary degree of neutrality, as seen in planetary magnetospheres $[1,2]$ and in the laboratory $[3,4]$. This makes it a promising geometry for the creation and study of an unusual object of research: a magnetized low-energy electron-positron pair plasma [5-7]. A basic prerequisite for the creation of such a plasma is the development of techniques for charged-particle injection into a closed magnetic system and subsequent particle manipulation and confinement. In electron experiments with the levitated dipole experiment Ring Trap 1 (RT-1), turbulence-induced inward transport was observed, and confinement times of hundreds of seconds were achieved [8]. To date, positrons in dipole traps are much less abundant and thus cannot benefit from collective effects. A series of experiments in the single-particle regime - i.e. with negligible space charge effects - have been conducted with positrons and a permanent magnet configuration providing the magnetic dipole field [9-11]. Lossless injection of positrons into this trap has been realized [10]. Once in the trap, the positron motion can be described by the guiding center drift Hamiltonian [12] with an effective potential $e \phi+\mu B$ (with $\phi$ the electrostatic potential; $\mu=m v_{\perp}^{2} / 2 B$ an adiabatic invariant; $e, m$ and $v_{\perp}$ the positron charge, mass and velocity perpendicular to the magnetic field $\mathbf{B}$ with $|\mathbf{B}|=B$ ). The guiding centers of the gyrating positrons $(0.1-10 \mathrm{GHz})$ bounce parallel to the magnetic field lines between the magnet poles $(1-10 \mathrm{MHz})$, and in addition they drift toroidally $(10-100 \mathrm{kHz})$ due to gradient and curvature drifts [13]. When strong electric field asymmetries are present, injected positrons remain confined for the time of approximately one toroidal transit (18 $\mu \mathrm{s})$ [9]. Suppression of these asymmetries has been found to yield confinement times corresponding to several hundreds and thousands of toroidal revolutions [9, 14]. However, the factors limiting confinement — as well as the nature of the confinement — remained undetermined.

In this letter we report on and discuss confinement times in excess of $1 \mathrm{~s}$ (hundreds of thousands of toroidal precession times) for a low-energy positron cloud in a magnetic dipole field superimposed by an external electric field. We compare this to the confinement of a positron cloud with a similar initial radial distribution in the same magnetic field but without the electrostatic contribution to the effective potential. We use these results to discriminate between different loss channels for the trapped particles. Trajectory simulations on the single-particle level further support our interpretation.

Experiments were carried out at the open beam port of the NEutron-induced POsitron source MUniCh (NEPOMUC) at Heinz Maier-Leibnitz Zentrum (MLZ), Garching [16, 17] 


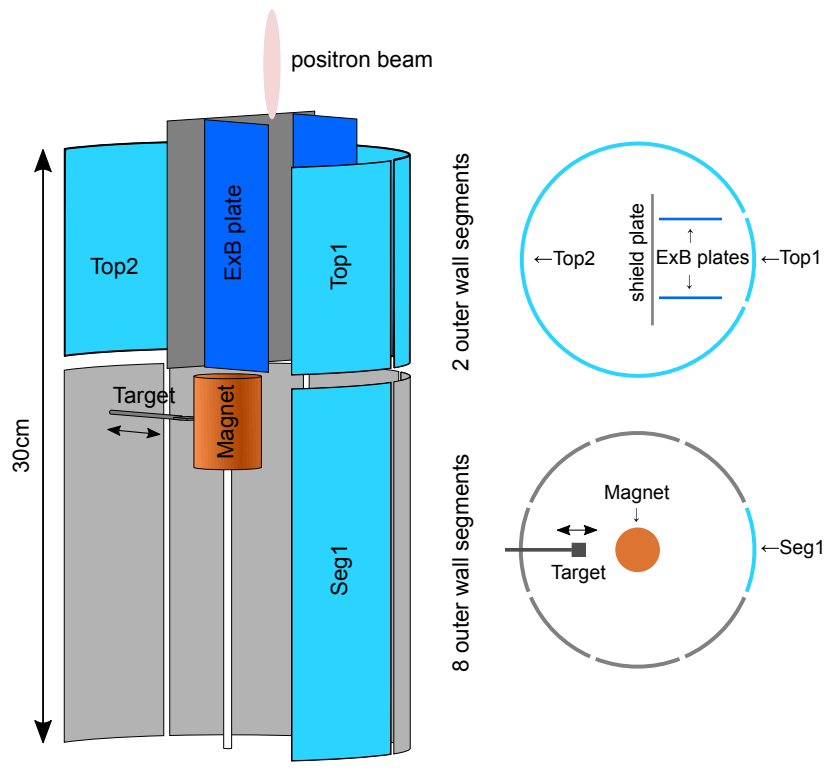

FIG. 1. Sketch (not all outer wall segments shown) and two cross sections of the trap showing the supported magnet and the electrode structure. The positron beam is injected from above by biasing the $\mathrm{E} \times \mathrm{B}$ plates and either electrodes Top1, Seg1 and the magnet (condition A) or electrodes Top1, Top2 and Seg1 [15]. For confinement measurements, the target probe was fully retracted from the trap.

with a prototype dipole trap: a central supported permanent magnet (field strength of $0.6 \mathrm{~T}$ at the poles) and a 10-segment cylindrical outer wall form the trap electrodes, as shown in Fig. 1. To guide positrons from the off-axis magnetic field lines into the confinement region of the magnet, a pair of electrodes is installed at the entrance port of the dipole trap; applying a bias to these plates induces an $\mathrm{E} \times \mathrm{B}$ drift across magnetic field lines. A grounded shield plate prevents this electric field from penetrating too deeply into the trap. Further details on the experimental setup can be found in $[9,10]$.

The experiment was operated at a residual gas pressure of $p=4.6 \cdot 10^{-6} \mathrm{~Pa}$. Via mass spectrometry, water vapor, nitrogen and molecular hydrogen have been identified as the main residual gas components. The DC remoderated positron beam had mean parallel and perpendicular energies of $E_{\|} \sim 5 \mathrm{eV}$ and $E_{\perp} \sim 1 \mathrm{eV}$, respectively, an energy spread of approximately $1 \mathrm{eV}[18]$ and a maximum flux of $2.4 \cdot 10^{7} e^{+} / \mathrm{s}$. The flux was determined with a charge-integrating amplifier connected to a copper plate that can be inserted to intersect the entire beam at the experiment's entrance.

For confinement measurements, the experiment was operated with repeated injection- 


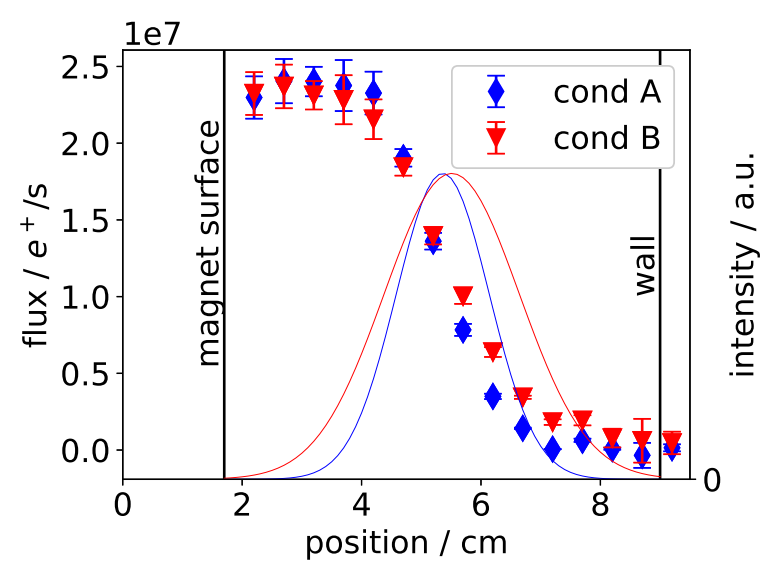

FIG. 2. Radial dependence of the positron flux in the magnetic dipole trap after half of a toroidal transit, measured with a charge-integrating amplifier connected to a radially insertable target probe. This is a spatially integrated measurement in the radial direction in the sense that all particles on field lines intersecting the target probe tip and its holder are measured at the same time. The data were fitted by complementary error functions (not shown), which gave for injection condition A (blue diamonds) a mean positron location of $(5.36 \pm 0.03) \mathrm{cm}$ and a spread of $(1.1 \pm$ $0.1) \mathrm{cm}$ and for injection condition B (red triangles) a mean of $(5.51 \pm 0.05) \mathrm{cm}$ and spread of $(1.6 \pm 0.1) \mathrm{cm}$. The thin lines show the radial positron distributions obtained from the derivatives of the respective fits.

hold-dump cycles (a timing diagram is shown in [15]) to obtain good signal-to-noise ratio. During the injection phase, the beam passed through an upstream cylindrical electrode and was transported across magnetic field lines into the trap by means of voltage biases applied to the $\mathrm{E} \times \mathrm{B}$ and other trap electrodes. We used two different configurations of bias voltages leading to lossless injection [10]: either two wall segments and the magnet (condition A) or three wall segments (condition B) were biased to a positive potential; the remaining trap electrodes were kept grounded. Both settings led to a similar radial distribution of the positron cloud in the magnetic dipole trap after half of a toroidal transit (Fig. 2). Due to the perturbing effect of the ExB plate biases, which leads to a loss of the trap content after approximately one toroidal transit, the number of trapped positrons amounts to a few hundred per cycle.

During the hold phase, the beam was blocked by biasing the upstream electrode; the 
E $\times$ B plates and all trap electrodes except the magnet were grounded. After a variable hold time $\Delta t$, the trap content was dumped by rebiasing the $\mathrm{E} \times \mathrm{B}$ and trap electrodes, which is known to cause a loss of confinement within microseconds [9]. The resulting annihilation radiation was recorded with a $B G O$ detector $(\mathrm{d}=22 \mathrm{~mm}, \mathrm{l}=25 \mathrm{~mm}$, from Korth Kristalle, with Hamamatsu type H10425 photomultiplier) with an uncollimated view onto the dipole trap. For each $\Delta t$, this measurement sequence was repeated 2048 times for condition $\mathrm{A}$ and 1920 times for condition B, and the respective annihilation signals were accumulated.

The background-subtracted data have been fitted by a single exponential without offset. For condition A, two independent data sets have been recorded and combined into a third, yielding $\tau_{A}=\{1082 \pm 154,1345 \pm 175,1492 \pm 126\} \mathrm{ms}$. For condition B, three independent data sets, requiring different data reconstruction algorithms (see [15]), have been recorded and combined into ten data sets, yielding confinement times of $\tau_{B} \in\{0.16 \pm 0.01,0.28 \pm$ 0.04\}. Examples of background-subtracted confinement curves for conditions A and B, respectively, are shown in Fig 3. Despite the similar initial radial distribution of positrons, the confinement times between the two conditions differ significantly. The main difference is the magnet bias during the hold phase (condition A: $V_{\text {mag }}=8 \mathrm{~V}>E_{e^{+}} / e$, condition B: $V_{\text {mag }}=0 \mathrm{~V}<E_{e^{+}} / e$, with $E_{e^{+}}$the positron kinetic energy). In condition A, application of the positive magnet bias adds a sufficiently large electrostatic trapping potential $e \phi$ to $\mu \mathrm{B}$. This confines nearly all positrons, not just those that have enough perpendicular velocity to be trapped by the $\mu \mathrm{B}$ potential created by the magnetic mirror of the dipole alone.

In the following, we discuss potential loss mechanisms for the positrons and their dependence on the experimental conditions. Generally, collisions with neutral species in the residual gas and asymmetries in the electric or magnetic fields are factors that can limit confinement. We find that collisions alone explain our experimental results reasonably well. There are several types of positron-neutral collisions: elastic collisions; inelastic collisions leading to some form of excitation (a change in rotational, vibrational or electronic state), ionization or positronium formation; and direct annihilation. Additional information on these processes is given in [15]. Due to the large mass difference between neutrals and positrons, elastic scattering changes mainly the positrons' pitch angle $\tan \theta=v_{\perp} / v_{\|}$but barely affects their kinetic energy. Ro-vibrational excitations usually have cross sections that are much smaller than the total cross section [19-25] and are thus considered negligible. The other three inelastic processes typically require a higher impact energy than $6 \mathrm{eV}$ 

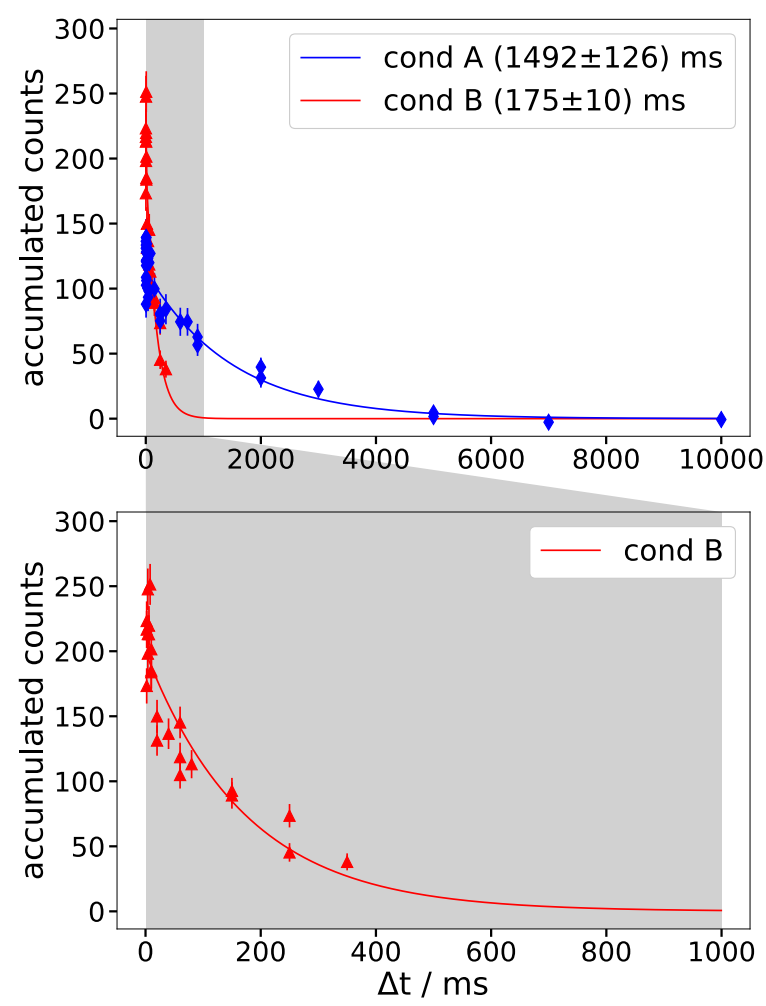

FIG. 3. The top panel shows one example of the accumulated positron annihilation counts vs. hold time $\Delta t$ for each of the two different settings of bias voltages of the trap: A (blue diamonds) and B (red triangles), see text for details. Solid lines give an exponential fit to the respective data. For condition B, the bottom panel gives an augmented view of the gray area in the top panel.

[26] or yield cross sections that are small (as in the case of water vapor). If direct annihilation was the dominant loss mechanism, lifetimes of several thousands of seconds [27] would be expected, which is much longer than the observed confinement times. Thus, pitch angle scattering is expected to be the dominant process.

The residual gas pressure in the experiment corresponds to a mean time between collisions of $1 / \nu_{\text {coll }}=1 /\left(n \sigma v_{\|}\right) \gtrsim 7 \mathrm{~ms}$, where $\sigma \sim 9.8 \cdot 10^{-20} \mathrm{~m}^{2}$ is the total cross section for collisions with water molecules at $6 \mathrm{eV}$ [22]. By comparing the collision time to the median confinement times $\tau_{A}=1345 \mathrm{~ms}$ and $\tau_{B}=174 \mathrm{~ms}$, we find they correspond to 192 and 25 collision events, respectively. Since only a few hundred positrons - an ensemble with negligible space charge - are trapped per cycle in our experiment, single-particle simulations were used. We investigated the influence of pitch angle scattering off neutrals on the location of 
particle losses while suppressing (condition A) or including (condition B) parallel losses onto the magnet poles via the electrostatic bias on the magnet case. The experiment was modeled in the trajectory simulation code AlGeoJ, described elsewhere [28]. The electric potential in these simulations has been calculated in a realistic geometry using the SIMION code [29]. As a benchmark, a particle distribution with the beam parameters described above was initialized in the beam line and injected into the trap, producing a radial distribution of particle orbits at the target probe position consistent with the experimental results in Fig. 2. In the experiment, the switch from the injection phase to the confinement phase occurs by ramping the potentials on the trap and $\mathrm{E} \times \mathrm{B}$ electrodes to ground in about $1 \mu \mathrm{s}$ (simultaneous with switching off the incoming positron beam by blocking it electrostatically further upstream). To model this process in the simulations, the particle distribution was initialized as before and the electrode biases were ramped linearly to ground potential in the course of also $1 \mu \mathrm{s}$. In order to represent particles at different locations in space during this ramp-down of the injection, particles are initialized from the same position but the start of the ramp-down was - for each particle — randomly chosen in the interval from zero to $20 \mu$ s after initialization. The confinement phase was then simulated using a simple collision model with an enhanced collision frequency of $2 \mathrm{MHz}$ in order to yield a reasonable computation time. With this enhancement, the collision frequency is still lower than the bounce and cyclotron frequency and should thus represent the same transport regime. At each collision event, the direction of the velocity vector was randomized. To correct for the enhanced ratio of collision to toroidal drift frequency, particles on orbits intersecting any trap electrode or the magnet during the remaining toroidal transit were removed from the simulation and the location of the loss was recorded.

Depending on the magnet bias, and thus the effective potential, we observe two qualitatively different effects in the simulations. When the magnet bias exceeds the particles' mean energy, the trapping well is sufficiently deep and parallel losses onto the magnet surface are prohibited for the majority of the particles. During approximately 560 scattering events, the particles are radially transported outwards until they reach orbits intersecting the shield plate or one of the $\mathrm{E} \times \mathrm{B}$ plates (see diagram in [15]) where they are lost, see Fig. 4a. In contrast, when no magnet bias is applied, the trapping potential is rather shallow and parallel losses are likely. Thus, most of the particles undergo little radial transport in about 28 collisions before being lost onto the magnet, see Fig. 4b. For both conditions A and B, the 

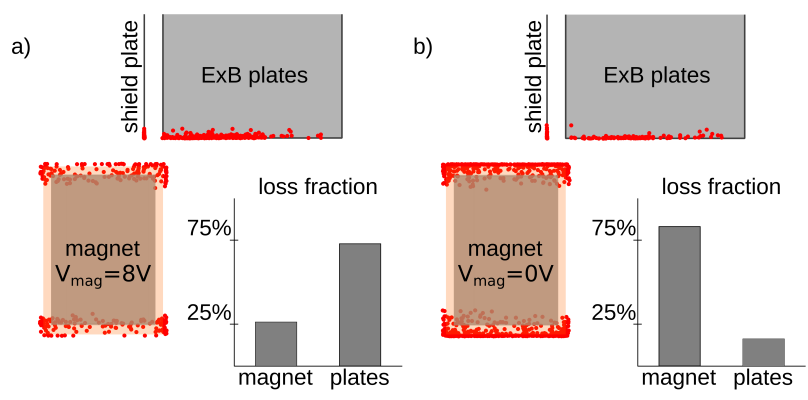

FIG. 4. Results of the confinement simulations for 10000 particles for conditions A and B (shown in panels a and b), respectively. The loss positions of particles on various electrodes are shown by red dots. The corresponding bar graphs give statistical information on the loss positions.

simulated number of collision events has the same order of magnitude as in the experiment. Their ratio (560:28) differs from the experimental case (192:25) by a factor of three. Two simplyfied approximations that do not capture the entire physics of the system might cause this discrepancy, mainly seen for condition A: first, the single exponential fits and second, the incomplete scattering model. The current body of data are not yet sufficient to make multi-parameter fits particularly meaningful (as the uncertainties are then very large). In upcoming work, we hope to improve the statistics of the data, upgrade the simulations to a more sophisticated scattering model, and explore fits with additional parameters. Nevertheless, the qualitative change in confinement seen in both experiment and simulation between conditions $\mathrm{A}$ and $\mathrm{B}$ leads us to conclude that pitch angle scattering in conjunction with the effective potential determined by the magnet bias reasonably explains the observed difference.

In summary, for the first time, to the best of our knowledge, we have confined positron clouds in a magnetic dipole field for longer than one second. This corresponds to many tens of pitch angle scattering events, and hundreds of thousands of toroidal precession times. This was achieved when applying a positive (repulsive) electrostatic bias to the magnet, which suppresses parallel losses onto the magnet surface. By improving the vacuum conditions a prolongation of the confinement is expected for future experiments in the described trap. Beyond that, these findings confirm our choice of a magnetic dipole geometry using a levitated current loop for future electron-positron pair plasma experiments, as the main loss channel identified here will be absent in this planned set-up.

This project has received funding from the European Research Council (ERC) under the 
European Union's Horizon 2020 research and innovation programme under grant agreement No 741322. The authors thank the DFG (No. Hu 978/15-1, Sa 2788/2-1), JSPS KAKENHI (No. 25707043, 16KK0094) and the Helmholtz Postdoc Programme (EVS) for funding. This work is based upon experiments performed at the NEPOMUC instrument operated by FRM II at the Heinz Maier-Leibnitz Zentrum (MLZ), Garching, Germany.

* juliane.stanja@ipp.mpg.de

[1] H. Alfvén, Cosmical Electrodynamics (Oxford University Press, 1950).

[2] J. A. Van Allen, Discovery of the Magnetosphere (History of Geophysics), Vol. 7 (American Geophysical Union, 1997).

[3] Z. Yoshida, H. Saitoh, J. Morikawa, Y. Yano, S. Watanabe, and Y. Ogawa, Phys. Rev. Lett. 104, 235004 (2010).

[4] A. C. Boxer, R. Bergmann, J. L. Ellsworth, D. T. Garnier, J. Kesner, M. E. Mauel, and P. Woskov, Nature Physics 6, 207 (2010).

[5] T. Sunn Pedersen, J. R. Danielson, C. Hugenschmidt, G. Marx, X. Sarasola, F. Schauer, L. Schweikhard, C. M. Surko, and E. Winkler, New J. Phys. 14, 035010 (2012).

[6] P. Helander, Phys. Rev. Lett. 113, 135003 (2014).

[7] P. Helander and J. W. Connor, J. Plasma Phys. 82, 905820301 (2016).

[8] H. Saitoh, Z. Yoshida, J. Morikawa, M. Furukawa, Y. Yano, Y. Kawai, M. Kobayashi, G. Vogel, and H. Mikami, Physics of Plasmas 18, 056102 (2011).

[9] H. Saitoh, J. Stanja, E. V. Stenson, U. Hergenhahn, H. Niemann, T. Sunn Pedersen, M. R. Stoneking, C. Piochacz, and C. Hugenschmidt, New Journal of Physics 17, 103038 (2015).

[10] E. V. Stenson, U. Hergenhahn, S. Nissl, J. Horn-Stanja, H. Saitoh, M. Singer, J. R. Danielson, M. R. Stoneking, M. Dickmann, C. Hugenschmidt, and T. Sunn Pedersen, (2018), accepted by PRL.

[11] H. Saitoh, J. Horn-Stanja, S. Nißl, E. V. Stenson, U. Hergenhahn, T. Sunn Pedersen, M. Singer, M. Dickmann, C. Hugenschmidt, M. R. Stoneking, J. R. Danielson, and C. M. Surko, AIP Conference Proceedings 1928, 020013 (2018).

[12] A. H. Boozer, Phys. Fluids 27, 2441 (1984).

[13] A. J. Dragt, Reviews of Geophysics 3, 255 (1965). 
[14] U. Hergenhahn, J. Horn-Stanja, S. Nißl, T. Sunn Pederesen, H. Saitoh, E. V. Stenson, M. R. Stoneking, M. Dickmann, C. Hugenschmidt, M. Singer, J. R. Danielson, and C. M. Surko, AIP Conference Proceedings 1928, 020004 (2018).

[15] See Supplemental Material at [URL will be inserted by publisher] for further details on the experiment, analysis and positron interactions with neutrals.

[16] C. Hugenschmidt, C. Piochacz, M. Reiner, and K. Schreckenbach, New J. Phys 14, 055027 (2012).

[17] C. Hugenschmidt, H. Ceeh, T. Gigl, F. Lippert, C. Piochacz, M. Reiner, K. Schreckenbach, S. Vohburger, J. Weber, and S. Zimnik, J. Phys.: Conf. Ser. 505, 012029 (2014).

[18] J. Stanja, U. Hergenhahn, H. Niemann, N. Paschkowski, T. Sunn Pedersen, H. Saitoh, E. V. Stenson, M. R. Stoneking, C. Hugenschmidt, and C. Piochacz, Nucl. Instr. and Meth. in Phys. Res. A 827, 52 (2016).

[19] S. Zhou, H. Li, W. E. Kauppila, C. K. Kwan, and T. S. Stein, Phys. Rev. A 55, 361 (1997).

[20] K. R. Hoffman, M. S. Dababneh, Y.-F. Hsieh, W. E. Kauppila, V. Pol, J. H. Smart, and T. S. Stein, Phys. Rev. A 25, 1393 (1982).

[21] J. P. Sullivan, S. J. Gilbert, J. P. Marler, L. D. Barnes, S. J. Buckman, and C. M. Surko, Nucl. Instr. and Meth. in Phys. Res. B 192, 3 (2002).

[22] A. Zecca, D. Sanyal, M. Chakrabarti, and M. J. Brunger, J. Phys. B: At. Mol. Opt. Phys. 39, 1597 (2006).

[23] T. Nishimura and F. A. Gianturco, Eur. Phys. J. D 33, 221 (2005).

[24] G. Karwasz, D. Pliszka, A. Zecca, and R. Brusa, J. Phys. B: At. Mol. Opt. Phys. 38, 1 (2005).

[25] F. A. Gianturco and T. Mukherjee, Phys. Rev. A 55, 1044 (1997).

[26] "NIST Chemistry WebBook," http://webbook.nist.gov, accessed: 13.11.2017.

[27] C. M. Surko, G. F. Gribakin, and S. J. Buckman, J. Phys. B: At. Mol. Opt. Phys. 38, R57 (2005).

[28] S. Nissl, Numerical Investigations into Injection and confinement of Single Particles in a Magnetic Dipole Trap, Master's thesis, Technische Universität München (2018).

[29] D. Manura and D. A. Dahl, SIMION ${ }^{\circledR} 8.0$ User Manual (Scientific Instrument Services, Inc. Ringoes, NJ 08551, 2008). 